\title{
TRABAJO Y PARTO PREMATUROS
}

\section{Doctor Jorge Villarreal Mejía (*)}

Introducción.-El trabajo prematuro del parto y los múltiples problemas concomitantes con el nacimiento del niño prematuro, han sido el tema de un gran número de publicaciones en la literatura médica durante los últimos cinco años.

Tres datos estadísticos brevemente nos ponen de presente la importancia de este tema desde el punto de vista médico-social:

1. El parto prematuro está catalogado como octavo entre todas las causas de muerte.

2. Las muertes de fetos viables prematuros constituyen el $10 \%$ de todas las defunciones en los Estados Unidos de América.

3. Mientras solamente $6 \%$ de todos los nacimientos son prematuros, la mortalidad en este grupo es veintiséis veces más grance que en niños nacidos a término.

Este trabajo presentará en su primera parte una actualización de los conceptos más recientes sobre el tema. La segunda parte estará dedicada a la revisión estadística de 215 nacimientos prematuros consecutivos sobre un período de dos años en el Centro Médico de la Universidad de Kansas, Estados Unidos de América.

Definición.-La definición de trabajo prematuro tiene que ser necesariamente correlacionada con la definición de parto y nacimiento prematuros. Según la mayoría de los autores, el niño prematuro es aquel que pesa 2.500 gramos o menos cuando nace. El término "prematuro" es por lo tanto engañoso, ya que la duración cel embarazo no entra en la definición. Prematurez, según este criterio, puede resultar de una terminación temprana del embara-

(*) Jefe de Residentes de Obstetricia y Ginecología. Centro Médico de la Universidad de Kansas, Estados Unidos de América. 1958. Residente Instructor del Departamento de Ginecología del Hospital de San Juan de Dios. Bogotá. 1959. 
zo, así planeada o por accidente, o por otra parte, ser la consecuencia de un erecimiento fetal lento, terminando en un niño de bajo peso cerca o a término.

Un criterio un poco más estricto pero desafortunadamente no seguido universalmente, es el usado en algunos hospitales universitarios de Norteamérica, donde se clasifican como prematuros a aquellos niños que llenan al menos dos de las tres condiciones siguientes: período de gestación de 28 a 37 semanas; una longitud entre 35 y 47 centímetros, y un peso de 1.000 a 2.499 gramos.

Frecuencia.-La frecuencia de trabajo y parto prematurcs $\unlhd a$ sido anotada por varios autores desde 3\% (10) hasta 12\% (12). La cifra reconocida como más comúnmente aceptada es probablemente entre 6 y $7 \%$.

A pesar de un progreso general en el manejo de las causas conocidas de prematurez, y los avances en el desarrollo de una actitud más racional en la prevención del trabajo prematuro, la frecuencia de nacimientos prematuros ha sufrido muy pequeña fluctuación a través de los años. La paridad materna juega un papel definitivo en esta entidad; la incidencia de terminación temprana del embarazo es de $8.3 \%$ en primíparas y solo $5.6 \%$ en multíparas. Se sabe igualmente que existe una mayor frecuencia ae prematurez en madres jóvenes y sin importar la edad de la madre, la incidencia de partos prematuros es mayor cuando el intervalo entre nacimientos es dos años o menos (7). Mientras que algunos estudios reportan una doble frecuencia de partos prematuros en pacientes sin ningún cuiclado prenatal (7), otros recalcan el hecho de que pacientes con un bajo nivel de vida, aun recibiendo adecuado cuidado prenatal, presentan un índice mucho más alto de prematurez. Este hecho puede ser substanciado por las ratas comparativas de partos prematuros, de $4.5 \%$ en pacientes privadas y de $11 \%$ en pacientes de caridad, según fue reportado en las series del doctor N. J. Eastman (34).

Varios autores de habla inglesa han recalcado la mayor frecuencia de niños prematuros nacidos de madres que viven en condiciones de hacinamiento y de aquellas que tienen embarazos ilícitos pero han sido contradictorios en la significación de la influencia del trabajo manual y de las ocupaciones durante el embarazo. Es lógico, por otra parte, que madres pequeñas den a luz niños de bajo peso, pero esencialmente maduros.

Hay un punto de acuerdo general acerca de la frecuencia del parto prematuro y es su ocurrencia en primíparas jóvenes que es- 
tán definitivamente por debajo de su peso normal y quienes además desarrollan toxemia del embarazo.

Etiología.-Según la mayoría de los autores las causas del comienzo prematuro del trabajo no pueden ser determinadas en más del $40 \%$ de los casos. La etiología puede ser clasificada bajo tres principales encabezamientos: materna, fetal y placentaria.

Materna: Toxemia.

Sífilis.

Enfermedades pulmonares.

Pielitis.

Patología pélvica.

Cirugía pélvica previa.

Enfermedades cardiovasculares.

Fetal: Fetos muertos macerados.

Embarazo múltiple.

Anormalidades congénitas.

Hidramnios.

Placentaria: Placenta previa.

Desprendimiento prematuro de placenta.

Ruptura prematura de las membranas.

Toxemia.-La frecuencia de toxemia en madres de niños prematuros ies generalmente tres veces más grande que en los grupos de control.

Algunos estudios (5) mencionan el incremento en el índice de prematurez únicamente con las formas más avanzadas de toxemia, y mencionan también el hecho de que toxemia gravídica, asociada con hemorragia del tercer trimestre, coincide generalmente con la más alta frecuencia de partos prematuros. Comparativamente, la incidencia de partos prematuros en varios grados de toxemia fue como sigue, en un estudio publicado por Elizabeth Larson en 1953 (16).

\section{Frecuencia de prematurez en pacientes toxémicas}

$5 \%$ en pacientes normales.

$7 \%$ en pacientes con toxemia moderada.

$17 \%$ en pacientes con toxemia grave.

$38 \%$ en pacientes eclámpticas. 
Sífilis. - La sífilis fue encontrada como la causa más probable de prematurez en $2 \%$ de 940 prematuros consecutivos en un estudio intensivo de once años (10). En las mismas series se encontraron fibromas uterinos en $3.4 \%$ de los casos y una frecuencia de operaciones pélvicas previas de cirugía mayor de $7.7 \%$, como los dos únicos factores que pudieron ser relacionados con el comienzo del trabajo prematuro en dichas pacientes.

Placenta previa.-La incidencia de placenta previa en nacimientos prematuros ha sido reportada (7) como 5\%, y la ocurrencia de desprendimiento prematuro de la placenta $3 \%$ en contraste con 0.5 y 0.2 a $1 \%$, respectivamente, en la población general.

En cuanto a las complicaciones hemorrágicas del último trimestre del embarazo, Brown y colaboradores (5), informando sobre 13.000 nacimientos consecutivos, catalogan la frecuencia de prematurez en relación con la historia de hemorragia y el origen de ia misma, como aparece en el cuadro número 2.

\section{Hemorragia del tercer trimestre asociada con partos prematuros}

\begin{tabular}{|c|c|c|}
\hline No hubo hemorragia $\ldots \ldots \ldots \ldots$ & & caso \\
\hline Hemorragia mínima .. & 14 & $"$ \\
\hline Placenta previa .. & 30 & $"$ \\
\hline Desprendimiento placentario . . . . . & 53 & " \\
\hline Historia de hemorragia y trauma & 27 & " \\
\hline
\end{tabular}

\section{CUADRO NUNGERO 2}

La ruptura prematura de las membranas amnióticas se ha mirado siempre como un signo definitivamente correlacionado con trabajo y parto prematuros. La importancia de este accidente reside en su relación con una mortalidad fetal aumentada en prematuros, así como en niños a término, según fue descrito por Calkins (6), quien en largo estudio estadístico en el Centro Médico de la Universidad de Kansas, Estados Unidos de América, encontró que la mortalidad fetal es por lo menos tres veces más grande cuando el período de tiempo entre la ruptura de las memhranas y el parto es de más de 24 horas.

La frecuencia de ruptura prematura de las membranas en relación con parto prematuro en la misma, fue como sigue: 
Niños a término (primíparas y múltíparas) . . . $12.5 \%$ Prematuros en primíparas ............ 22.0\% Prematuros en multíparas . . . . . . . . . . . $32.0 \%$

El mecanismo por el cual la ruptura de las membranas trae consigo el comienzo del trabajo, es esencialmente el mismo en partos prematuros así como a término. Estudios con el Tocodinamómetro (26), han revelado que aunque las tensiones absolutas en los tejidos uterinos disminuyen algo después de la ruptura de las membranas, el principal efecto de este fenómeno es el aumentar la tensión dentro del fondo del útero en comparación con la que existe en el segmento bajo del mismo. Esto trae como consecuencia el desarreglo del balance que en condiciones normales previene al útero de desocupar sus contenidos y en este momento el esquema normal de contracciones uterinas queda establecido.

Tieche y colaboradores (30), estudiando las tres causas más comúnmente aceptadas de trabajo prematuro: toxemia, hemorragia tardía en el embarazo y ruptura prematura de las membranas, guardaron cuidadosos controles de los partos a término para cada uno de los grupos mencionados. Estos autores encontraron que de 57 casos de ruptura prematura de las membranas, 20 fueron prematuros y 37 fueron niños a término. Siendo así que ellos encontraron que $8.26 \%$ fue la incidencia de prematurez en el grupo general; ellos calculan que únicamente 5 , o sea $8.26 \%$ de 57 , podrían ser estadísticamente aceptados como cifra normal representativa en el grupo de prematuros y que por lo tanto los otros 15 casos debieron haber sido causados por la ruptura prematura de las membranas únicamente.

Usando el mismo criterio en las toxemias del embarazo asociadas con prematurez, ellos encontraron que únicamente 3 de cada 7 nacimientos prematuros pudieron ser culpados a esta complicación. La cifra correspondiente a la separación prematura y parcial de la placenta fue un aumento del $50 \%$ en partos prematuros. La última, aunque frecuente causa placentaria de trabajo prematuro que debemos mencionar, es la inserción marginal del cordón umbilical, que, de acuerdo con Brody y Frenkel (14), es acompañada por nacimiento prematuro en el $65 \%$ de los casos. El mecanismo de producción de niños prematuros sería en este caso la interferencia eventual con el abastecimiento de sangre al feto, posiblemente iniciando trabajo prematuro o produciendo un niño de bajo peso y de inadiecuada nutrición. 
La discusión sobre la etiología del trabajo y parto prematuros no podría ser completa si omitiéramos algunos comentarios acerca de la influencia de la nutrición y la dieta como factores causales.

La influencia de mala nutrición, dieta inadecuada y su coroiario, pacientes pobremente nutridas y muy por debajo de su peso normal, ha sido considerado por muchos como una de las principales causas de partos prematuros. De acuerdo con Tompkins (33), es un error el considerar "nutrición" únicamente en las bases de consumo alimenticio, pues mientras la dieta ayuda en el soporte de la nutrición, los dos términos no se deben considerar sinónimos. El mismo autor define nutrición como la suma de los procesos por los cuales el organismo absorbe y utiliza substancias esenciales para el soporte del metabolismo de las células que almacenan para uso futuro o usa inmediatamente para liberación de energía. También incluye la suma de las influencias que los ambientes intrínseco y extrínseco ejercen sobre estos procesos.

En sus muy bien documentadas series, Tompkins encontró un indice de trabajo prematuro $41 \%$ más bajo en un grupo con marsada mejoría en su nutrición; pero explícitamente declara que la dieta en el embarazo es solo secundaria en importancia a otras muchas condiciones que pueden influenciar la nutrición de cada paciente. Speert y colaboradores (29), en una revisión de la literatura sobre este tema en 1951, encontraron reportes contradictorios sobre la correlación de dieta y prematurez en periodos de guerra y también encontraron que dietas de hambre en ratas producían interferencia con el crecimiento fetal más bien que aumento en trabajo y nacimientos prematuros propiamente dichos. Los mismos autores reportaron el hecho de que determinaciones bioquímicas y hematológicas de varios principios alimenticios en mujeres embarazadas, no llegaron a revelar ninguna diferencia significante entre madres de prematuros y madres de niños a término.

Desde el punto de vista práctico, las curvas de aumento de peso durante el embarazo y el peso al comienzo del mismo son la guía más usada para la estimación de la nutrición. De acuerdo con la mayoría de los autores $(17,33)$ el esquema característico de peso en casos de trabajo prematuro, se establece con un bajo jeso inicial y el fallo de la enferma en aumentarlo a una rata aceptable durante los dos primeros trimestres. Este patrón es establecido muy temprano durante el embarazo y para el fin del sexto mes ya no puede ser alterado. 


\section{Peso de las madres y ratas comparativas de prematurez}

Peso normal o sobre-peso . . . . . . . . . . . . . . . $5 \%$

Más de $5 \%$ de peso por debajo del normal . . . . . . $10 \%$

Más de $20 \%$ de peso por debajo del normal . . . . 20\%

El hecho de que varios grados de anemia al comienzo del embarazo se encuentren ocasionalmente asociados con índices aumentados de trabajo prematuro, ha sido mencionado por algunos investigadores de este tema (17, 33).

Para cerrar este capítulo sobre los factores nutricionales en la etiología de trabajo y parto prematuros, debemos mencionar el trabajo de Mundall (21), quien afirma que "existe una evidencia sugestiva, aunque limitada, de la posible relación entre alergias alimenticias y contracciones prematuras del útero gestante". Los alimentos frecuentemente inculpados son peces de agua salada, mariscos y la carne de cerdo. Los síntomas de este fenómeno alérgico son: dolor de espalda y de cabeza, palpitaciones, calambres uterinos, hemorragia y ocasionalmente trabajo activo. El tratamiento de elección aconsejado sería la administración de compuestos antihistamínicos por vía oral o intravenosa, seguidos por enemas de solución salina isotónica.

La investigación en el campo de trabajo prematuro del parto ha sido marcadamente escasa. Reynolds, Harris y Kaiser (27), midiendo las fuerzas uterinas durante el embarazo y el trabajo por medio del tokodinamómetro, mostraron que la fibra uterina sufre un proceso de maduración durante el embarazo, el cual es terminado en una fecha más temprana en el grupo de pacientes que luego entran en trabajo prematuro. Siendo asi que estas pacientes, comenzando temprano durante el embarazo, tienen una actividad irregular y aumentada del útero en comparación con sujetos controles, esto podría explicar los casos frecuentes en que mujeres por otra parte enteramente normales entran prematuramente en trabajo. Los mismos estudios de contracción uterina revelaron que el trabajo prematuro es caracterizado por una mayor actividad en el segmento medio e inferior que el presente en trabajo normal. Esto es particularmente cierto al comienzo del trabajo prematuro: el esquema, siendo similar al normal a medida que el trabajo progresa. Este factor no ha podido ser asociado con la patogénesis del trabajo prematuro.

Aarón y colaboradores (1), en un intento de investigar algunas de las causas menos conocidas de trabajo prematuro, organi- 
zaron una "clínica de salvación del embarazo" dedicada a estudiar parejas que habian tenido por lo menos dos embarazos sin fruto, terminados por cualquiera de estas cinco vías: a) abortos repetidos; b) nacimientos prematuros; c) nacidos muertos; d) muertes neonatales; e) niños congénitamente defectuosos. El estudio de estas parejas incluyó: 1) anormalidades anatómicas y funcionales del útero; 2) examen completo del sistema génito-urinario en el hombre; 3) evaluación genética; 4) investigaciones nutricionales; 5) estado sociológico; 6) test endocrinos, y 7) tests psicológicos. El material usado fueron 52 parejas que habían tenido 191 embarazos y producido 18 niños vivos, 12 a término y 6 prematuros. Estos autores encontraron que los factores ginecológicos constitucionales, incidencia familiar, endocrinopatías, factores psicológicos y nutricionales no contribuyen substancialmente a las causas del aborto habitual, prematuridad, nacidos muertos, etc., pero en cambio las anormalidades uterinas anatómicas y los fallos endometriales parecen jugar un papel importante en el 60\% de trabajos y nacimientos prematuros.

Mortalidad fetal.-Aunque la incidencia de prematurez no ha variado apreciablemente a través de los años, la rata de mortalicad de los niños prematuros ha disminuído persistentemente durante los últimos decenios, debido a nacimientos menos traumátıcos, anestesia más segura y mejor cuidado pediátrico.

En un estudio de diez años publicado en 1952, Dana (10) reportó una mortalidad fetal bruta de $41 \%$ en 1933, en contraste con $23 \%$ en 1943.

\section{Mortalidad fetal en prematuros}

Dentro de las primeras 24 horas post-parto . . . . . . 57\%

Dentro de la primera semana post-parto ... . . . $33 \%$

Si hubo toxemia gravídica asociada . . . . . . . . . $37 \%$

En caso de presentarse patología placentaria .. . . $63 \%$

\section{CUADRO NUMERO 3}

Se sabe que la mayoría de las muertes neonatales ocurren en el primer día y la mayoría de ellas son de niños prematuros.

Hemorragias durante el embarazo parecen tener una gran importancia como causa asociada de mortalidad fetal, según mostó Schoeneck (28), quien en un estudio estadístico de 3.235 naci- 
mientos prematuros sobre un período de cinco años, encontró que $26.5 \%$ de las madres de niños prematuros que murieron habían presentado hemorragia vaginal en alguna época durante el embarazo.

El peso del recién nacido y su condición general al momento del parto, parecen influenciar definitivamente las ratas de mortalidad, según mostró Whitner (32) en un estudio de 458 nacimientos prematuros consecutivos.

\section{Mortalidad fetal en prematuros}

$80 \%$ en los niños por debajo de dos libras y media.

$1 \%$ en los niños por encima de cinco libras.

$15 \%$ si las condiciones generales del niño son buenas al nacer. $49 \%$ si las condiciones generales del niño son malas al nacer.

\section{CUADRO NUMERO 4}

La asociación de peso por debajo de cuatro libras, condición pobre al nacer y una historia de hemorragia materna prenatal, diron en las series de Whitner una mortalidad del $82 \%$.

Uno de los hallazgos más frecuentes en niños prematuros que mueren es el de membranas hialinas en los pulmones. Greenhill asegura que el $50 \%$ de los niños que mueren después de nacimientos prematuros muestran membranas hialinas en la autopsia, en contraste con únicamente $25 \%$ de los niños que mueren después de partos a término. El mismo autor menciona que raramente se encuentran membranas hialinas si la muerte ocurre menos de una hora o más de cinco días después del nacimiento.

Profilaxis deî trabajo prematuro.-En relación con la prevención del trabajo prematuro, muy poco se ha obtenido fuera del evitar o tratar en sus faces iniciales las condiciones generalmente reconocidas como causantes de trabajo y parto prematuros. Smith y Smith (31), en un análisis estadílstico de 76 pacientes que havían tenido partos prematuros repetidos, reportaron una disminución en el índice de prematurez cuando las mismas pacientes es: uvieron bajo tratamiento con estrógenos en embarazos subsiguientes. Estos autores aseguran que los niños de madres tratadas con stilbestrol son excepcionalmente grandes y pesados para su edad gestacional, y que no importa cuál fue su tamaño, muchos de ellos vivieron. Fergusson (13), del Charity Hospital de 
Louisiana, en un trabajo muy bien documentado, dio stilbestrol en dosis crecientes, hasta un máximo de 137.5 miligramos diarios a 190 enfermas y solo placebos a 203 controles. Los resultados obtenicos no revelaron absolutamente ninguna mejoría en los índices de vrematurez o en el peso de los niños en el grupo de stilbestrol en -omparación con los controles. De acuerdo con Nesbitt (22), la influencia de cirugía abdominal como causa de trabajo prematuro sería mucho menos marcada si las operaciones electivas se hicieran entre las 18 y 20 semanas de embarazo.

Manejo del trabajo prematuro.-Antes de entrar en los detailes del manejo del trabajo prematuro, debemos dar alguna consideración a la ruptura espontánea y' prematura de las membranas amnióticas. Este accidente ocurre, según la mayoría de los autores, en un $17 \%$ de los casos como preludio de trabajo prematuro.

El manejo de la ruptura prematura de las membranas consis-te, primern que todo, en hospitalización inmediata para observa cióli adecuada de la paciente. Tratamiento con antibióticos de amplio espectro se debe comenzar inmediatamente y continuarlo por 24 horas, pero principalmente debe hacerse al comienzo del trabajo activo, puesto que las bacterias del tracto genital bajo tienden a irse hacia arriba al comienzo de la actividad uterina.

Eastman (12) dice que el principal peligro potencial después de la ruptura prematura de las membranas es la infección por el coito, y si éste puede evitarse, la enferma puede ser enviada a su casa. De acuerdo con Calkins (6), si la infección no se presenta dentro de 72 horas después de la ruptura, muy probablemente nunca ocurrirá. Asegura el mismo autor que inducción del trabajo debido a ruptura prematura de las membranas solo se justifica en casos muy bien seleccionados.

La duración del trabajo del parto es reconocidamente más corta en trabajos prematuros que en pacientes a término. 


\section{Duración del trabajo del parto en nacimientos prematuros}

\section{Primíparas:}

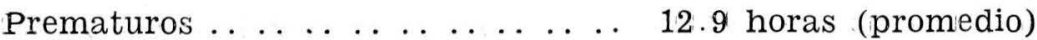

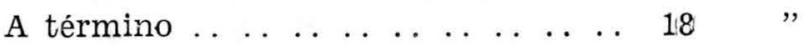

\section{Multíparias:}

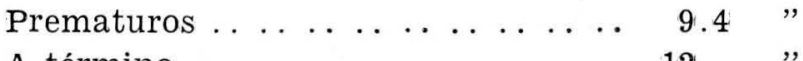

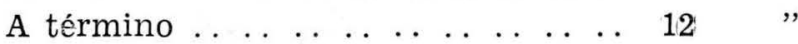

\section{Trabajo prolongado:}

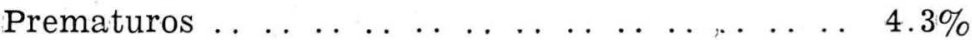

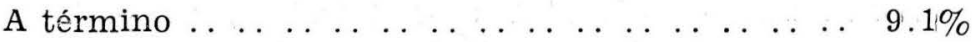

\section{CUADRO NUMERO 5}

Una vez que el trabajo prematuro irreversible ha sido establecido, la conducta a seguir puede ser esquematizada de la manera siguicnte:

1. Evitar los calmantes, excepto en dosis mínimas.

2. Dar una compañía adecuada a la paciente.

3. Seguir el progreso del trabajo por observación general y por el carácter de las contracciones, más bien que por exámenes frecuentes rectales o vaginales.

4. Nutrición adecuada de la enferma en trabajo, con generosa administración de líquidos por vía oral o intravenosa.

Desde el descubrimiento de la hormona relaxante uterina por Hisaw en 1926, numerosos trabajos de experimentación animal sobre esta hormona han sido publicados, pero ha sido únicamente durante los últimos cinco años que estudios en la raza humana han sido llevados a cabo en el uso de esta hormona para detener el trabajo prematuro del parto $(18,19)$. Los resultados de estos estudios han sido muy contradictorios hasta la fecha.

Manejo del parto prematura.-Cinco puntos principales deben tenerse en cuenta en el manejo del parto prematuro:

1. Un pediatra debe estar presente en la sala de partos.

2. Usese anestesia local o regional.

3. Episiotomía amplia.

4. Inhalaciones de $100 \%$ de oxígeno en el período de desprendimiento. 
5. Cortar el cordón umbilical después de que las pulsaciones hayan cesado.

La selección de la anestesia es de gran importancia, y muchos autores consideran que el óxido nitroso es la mejor posibilidad si no se pueden usar los bloqueos regionales o locales. El óxido nitroso alivia el dolor de la madre, sin producir pérdida de' la conciencia sin asfixiar ni a la madre ni al niño, y sin interferir con la iniciación espontánea de la respiración del niño después del nacimiento.

Universalmente está acordado que las madres toxémicas deben ser ayudadas con inducción o dar a luz por operación cesárea entre la 36ạ y 37ạ semanas de gestación. El alumbramiento de ıas pacientes toxémicas por medio de la cesárea, está muy lejos de ser la respuesta ideal a este problema, según demostró Dana, en cuyas series la mortalidad fetal total para operación resárea fue de $47 \%$, en contraste con $29 \%$ para los nacimientos espontáneos. Esto se explica en parte por la más alta incidencia de graves complicaciones maternas que conducen a la práctica de dicha operación.

Las mismais series nombradas más arriba revelaron un aumento en la morbiliaad materna de $9.4 \%$ en el grupo total de pacientes, hasta $15.7 \%$ en las madres de niños prematuros. Presentaciones fetales anormales son generalmente reconocidas como de mucha más frecuente ocurrencia en los partos prematuros; la presentación de nalgas aumentó cuatro veces en frecuencia y la presentación de cara tres veces.

El niño prematuro.-El prematuro, según la descripción de Greenhill, posee las siguientes características:

Cuerpo generalmente pequeño y encanijado.

Piel suave, delgada y en pliegues de color rojo vivo.

Tejido adiposo escaso.

Lanugo abundante, especialmente en las superficies de extensión.

Cráneo con fontanelas abiertas y suturas prominentes.

Uñas cortas.

Llanto monótono y débil.

Temperatura irregular por debajo de lo normal.

Orina escasa.

El prematuro debe tener una vía respiratoria limpia, oxigeuación adecuađa, tibieza ambiental y tan poco manoseo como sea cosible. Las drogas de estimulación cardíaca o respiratoria deben 
ser totalmente evitadas. El moco debe ser removido de las vías respiratorias únicamente por medio de un catéter blando. La costumbre de administrar vitamina $\mathrm{K}$ a los recién nacidos prematuros, aunque sugerida por algunos autores (32), es de un valor dudoso.

Pronóstico del niño prematuro--Es generalmente reconocido que los niños prematuros tienen más posibilidades de nacer con defectos físicos o mentales debido a malformaciones congénitas y que algunos de esos defectos pueden ser modificados mediante diagnóstico oportuno y tratamiento quirúrgico experto de las malformaciones.

También, de acuerdo con Crosse (8), los recién nacidos prematuros son más propensos a desarrollar alteraciones mentales o físicas como resultado de complicaciones durante el trabajo del parto o el período neonatal, algunas previsibles en su totalidad, como la fibroplasia del cristalino ocular, mientras que otras solo reJuctibles mediante cuidados especializados, como las lesiones producidas en partos traumáticos, asfixia e infecciones (17 A).

\section{Trabajo y parto prematuros.-Estudio estadístico}

(Centro Médico. Universidad de Kansas, Estados Unidos)

Material.-Este estudio comprendió un período de dos años (1955-1956), durante el cual hubo 215 nacimientos prematuros $y$ un total de nacimientos de 3.583 , lo que da una frecuencia de parto prematuro de $6.07 \%$.

La distribución por razas y categoría hospitalaria puede apreciarse en el cuadro número 6 :

\section{Parto prematuro en el Centro Médico.-Universidad de Kansas}

Distribución racial:

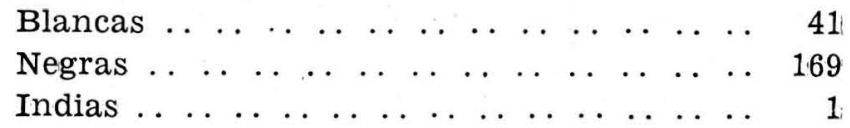

Clasificación hospitalaria:

Pacientes de caridad . . . . . . . . . . . . . . . . . 193

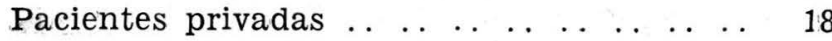


La distribución de las madres de niños prematuros según su edad, puede apreciarse en el cuadro número 7. El mayor grupo correspondió a aquellas madres entre los quince y veinte años de edad. En un estudio de recién nacidos prematuros reportado por Baird y Winnicott (2), un gran número de niños prematuros fueron comparados con niños a término como control. Ambos niños en cada pareja habían nacido en la misma semana, del mismo sexo, el mismo lugar, venían del mismo tipo de hogar y vivían en regiones similares. El estudio mostró que los niños prematuros tueron admítidos tres veces más al hospital durante los dos primeros años de vida, estuvieron tan atrasados en peso con respecto a sus controles, a los cuatro años, como al nacer y aproximadamente un $40 \%$ de ellos eliminaron sus defectos físicos antes de los cuatro años de edad.

Con el objeto de mejorar el pronóstico de los recién nacidos prematuros, varios hospitales universitarios de los Estados Unidos de Norteamérica organizaron hace varios años un plan para el cuidado controlado de los niños prematuros. Este es un esfuerzo conjunto del Departamento de Pediatría, el servicio de enfermeras y trabajadoras sociales para proveer al recién nacido prematuro con un cuidado adecuado en su casa después de ser dado de alta del hospital, descubrir y evitar enfermedades en el ambiente familiar y servir de consultor y consejero en todos los problemas sociales de la familia, que en una $u$ otra forma puedan amenazar la vida del recién nacido prematuro.

\section{Distribución de las madres de niños prematuros según su edad}

Menores de quince años: $0.9 \%, 4$ casos.

Quince a veinte años: $40.7 \%, 82$ casos.

Veintiuno a veinticinco años: $27.4 \%, 60$ casos.

Veintiséis a treinta años: $14.7 \%, 30$ casos.

Treinta y uno a treinta y cinco años: $7.1 \%, 12$ casos.

Mayores de treinta y cinco años: $9 \%, 15$ casos.

\section{CUADRO NUMERO 6}

El tiempo de gestación fue de 36 semanas en 27 de las 215 pacientes, lo cual constituye el grupo mayor de enfermas para una edad gestacional dada.

Una cuarta parte de las madres de niños prematuros en este estudio no recibió ningún cuidado prenatal. La mayoría, como 
puede apreciarse en el cuadro número 7, recibieron de uno a tres meses de control prenatal. La historia de partos prematuros previos no influyó apreciabiemente las ratas de prematurez, como puede verse en el cuadro número 8.

\section{Cuidado prenatal en madres de niños prematuros}

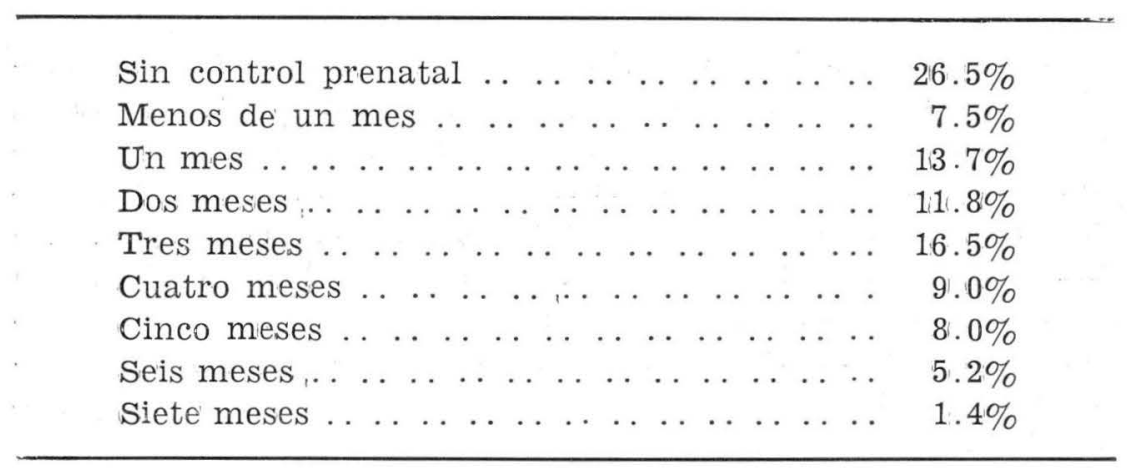

CUADRO NUMERO $\%$

Historia de prematurez en madres de niños prematuros

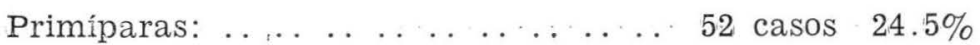
Multíparas:

Sin previos partos prematuros .. . . . 86 casos $40.0 \%$

1. parto prematuro previo ... . . . 47 " $42.2 \%$

2 partos prematuros previos . . . . . . 12 " . . $5.6 \%$

3 partos prematuros previos ....... . 7 " $3 \%$

4 partos prematuros previos ...... . . 2 " $20.9 \%$

5 partos prematuros previos ....... 4 . 4 " . $1.8 \%$

6 partos prematuros previos ....... 1 . $\quad$ " $0.4 \%$

\section{CUADRO NUMERO 8}

El grupo mayor de pacientes, al ser éstas agrupadas según su peso en relación con el promedio normal para su estatura, lo constituyó 24 enfermas, quienes estaban cinco libras por debajo de 10 normal. Las pacientes con sobrepeso formaron los grupos más pequeños, en contraste con aquellas con pesos por debajo de lo normal.

La frecuencia de diversos factores sanguíneos en las pacientes que tuvieron partos prematuros, puede apreciarse en la lista que sigue: 


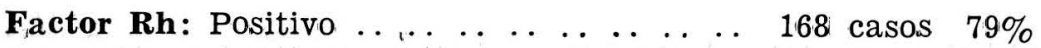

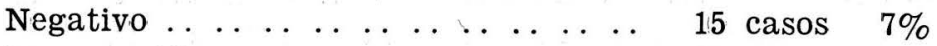

Desconocido . . . . . . . . . . . . . . . . . 28 casos $13 \%$

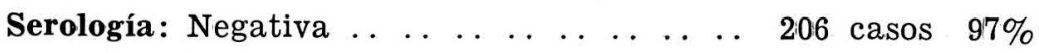

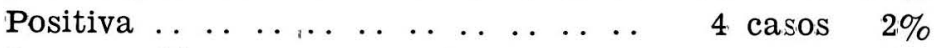

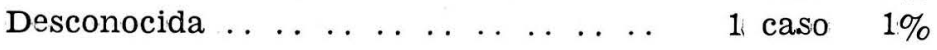

Los agentes analgésicos y anestésicos usados durante el trabajo y partos prematuros fueron, en la gran mayoría de los casos, ei óxido nitroso y los bloqueos regionales o locales. El óxido nitroso se administró en una concentración del 40 al 60\%, y únicamente durante las contracciones uterinas. IComo se puede apreciar en el cuadro número 9, durante el período de dilatación muchas pacientes no requirieron anestesia alguna. Es de anotarse igualmente la muy baja frecuencia con que agentes tan poderosos como la morfina y el demerol se usaron en estas series (Cuaclros números 9 y 10 ).

\section{Anestesia usada durante el periodo de dilatación}

$\begin{array}{llllllllllllllll}\text { Ninguna } & \ldots & \ldots & \ldots & \ldots & \ldots & \ldots & \ldots & \ldots & \ldots & \ldots & \ldots & \ldots & \ldots & 42.1 \%\end{array}$

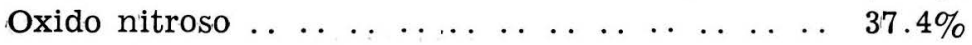

Anestesia espinal en "silla" . . . . . . . . . . . . . . $\quad 8.0 \%$

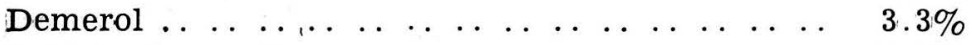

Sulfato de morfina . . . . . . . . . . . . . . . . . . $\quad 2.8 \%$

Oxido nitroso + anestesia en silla . ... . . . . $1.4 \%$

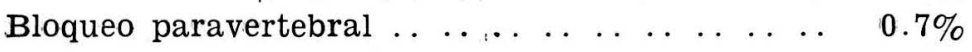

CUADRO NUMERO 9

Anestesia y analgesia usadas en partos prematuros

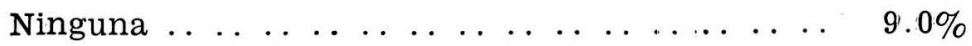

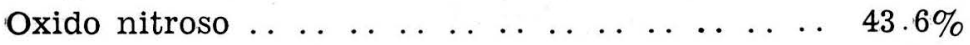

Oxido nitroso + bloqueo pudendo ........ . $13.7 \%$

Anestesia espinal en silla . . . . . . . . . . . . $10.4 \%$

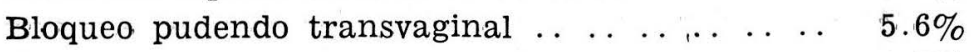

Oxido nitroso + trilene . . . . . . . . . . . . . . $4.7 \%$

Oxido nitroso + anestesia local . . . . . . . . . . $4.7 \%$

Oxido nitroso + anestesia espinal en silla .. ... $4.7 \%$ 
El tipo de parto en los nacimientos prematuros en el Hospital Universitario de Kansas, Estados Unidos de América, puede apreciarse en el cuadro número 11. Nótese la baja incidencia de partos intervenidos con fórceps, así como también la relativamente baja frecuencia de operaciones cesáreas. Pese a lo hoy día generalmente aconsejado por la mayoría de los autores, una gran proporción de pacientes no tuvieron episiotomía.

\section{Nacimientos prematuros. Tipo de płarto}

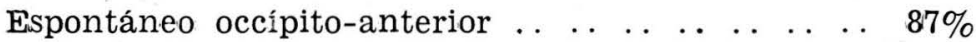

Espontáneo, posiciones posteriores . . . . . . . . . . $1 \%$

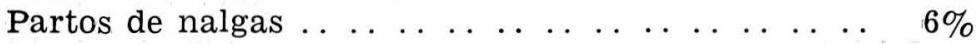

Partos con fórceps . . . . . . . . . . . . . . . . . . . . . . .

Operaciones cesáreas . . . . . . . . . . . . . . . $\quad 3 \%$

Partos en que se usó episiotomía . . . . . . . . . . . . $33 \%$

Partos en que no se usó episiotomía ... . . . . . . . $\quad 67 \%$

\section{CUADRO NUMERO 11}

La relación entre patología placentaria y tiempo de espera después de la ruptura prematura de las membranas amnióticas, puede apreciarse en el cuadro número 12 . Vemos que cuando el período de intervalo entre ruptura de membranas y nacimiento del niño fue de uno a tres días, la frecuencia de placentitis ascendió a $77 \%$. En contraste, cuando éste período de espera o intervalo fue de más de una semana, la frecuencia de placentitis fue de $25 \%$.

\section{Patología placentaria asociada con ruptura prematura de has membranas}

\section{Período de intervalo}

menos de un día, 8 casos .. ... 50\% revelaron placentitis 1. a 3 días, 18 casos ......... . 77\% revelaron placentitis 3 a 7 dias, 10 casos .. . . . . . . 50\% revelaron placentitis más de una semana .. ...... $25 \%$ revelaron placentitis 
Solamente ciento veinte de las placentas obtenidas de partos prematuros fueron sometidas a examen anatomopatológico. Aproximadamente la mitad de ellas no revelaron signo alguno de prematurez, y aproximadamente una tercera parte mostraron signos variables de infección secundaria (Cuadro № 13).

\section{Patología placentaria en partos prematuros}

Placenta inmadura . . . . . . . . . . . . . . . . . . . .

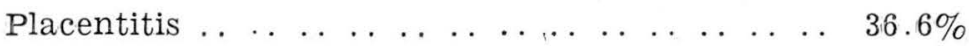

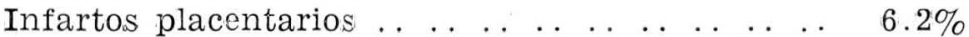

Infecciones del cordón umbilical . . . . . . . . . $3.3 \%$

Placenta circunvalata . . . . . . . . . . . . . . . . .

\section{CUADRO NUMERO 13}

\section{Comentarios}

La extensa literatura sobre el tema de trabajo y parto pre. maturos carece de uniformidad en el criterio usado para definiros, haciendo en esta forma más difícil la evacuación de los reción, haciendo en esta forma más difícil la evaluación de los resultados obtenidos en diferentes series. Para hacer este estudio aún más inexacto, la duración del embarazo ha sido excluída en el criterio de definición usado en la mayoría de los trabajos; por io tanto, clasificando como prematuros a recién nacidos cuyo bajo peso no es el resultado de prematurez propiamente dicha, sino la consecuencia de una interferencia con el crecimiento fetal normal. Speert y colaboradores (29), comunicaron sobre un estudio hecho por Bancroft, quien en 1947 encontró que 19\% de casos de prematurez inexplicables habian llegado realmente a las 40 semanas de gestación.

Desde un punto de vista esencialmente práctico, es sabido que mientras la prematurez está catalogada mundialmente como una de las principales causas de muerte, la cantidad de dinero empleado en la investigación de la prevención del trabajo y parto prematuros es muy pequeña en contraste con el costoso presupuesto de investigación para otras enfermedades de más nombre. Points (25), informando sobre este tema, dice que por cada muerte de un prematuro la cantidad de dinero de investigación gastado en cada muerte por enfermedades cardio-vasculares, cáncer y poliomielitis, es tres, ocho y medio y setenta y seis veces más grande, respectivamente. 


\section{CONCLUSIONES}

1. El estudio de trabajo y parto prematuros adelantará mucho el día que se llegue a un acuerdo universal en el criterio de definición.

2. Mientras que la incidencia de partos prematuros no ha cambiado apreciablemente a través de los años, la mortalidad fetal en este grupo ha venido disminuyendo de continuo.

3. Entre las causas de prematurez, la toxemia todavía ocupa un puesto prominente junto con la asociación de primiparidad y bajo peso.

4. Aunque el parto prematuro es usualmente fácil para la madre y el obstetra, el recién nacido puede, en cambio, ser fácilmente maltratado si las precauciones debidas son pasadas por alto.

5. El pronóstico del recién nacido prematuro depende en gran parte de su peso y de su condición general en el momento de nacer.

6. La frecuencia de trabajo y parto prematuros en el Centro Médico de la Universidad de Kansas, Estados Unidos de América, durante un período de dos años (1995-1956), estuvo de acuerdo con las cifras más frecuentemente citadas en la literatura.

7. La importancia de cuidado prenatal adecuado en la prevención de prematurez, fue claramente revelada en la ocurrencia de partos prematuros veinticinco veces más frecuentes en pacientes sin cuidado prenatal, que en aquellos que fueron seguidos por un obstetra desde muy temprano en su embarazo.

8. En este corto estudio la historia de trabajos prematuros anteriores fue encontrada sin ninguna correlación con la ocurrencia de los más recientes partos prematuros.

9. La incidencia de procesos inflamatorios después de la ruptura prematura de las membranas amnióticas fue más grande cuando este accidente ocurrió dentro de una semana antes del parto.

10. El hecho de que $12 \%$ de las placentas obtenidas en casos de parto prematuro en las series presentadas, fueron encontradas sin ninguna evidencia histológica de prematurez, hace pensar al que estudia este tema si este grupo no representa simplemente niños de bajo peso pero maduros o a término.

11. Soluciones adecuadas a los problemas arriba mencionados y a muchos otros sobre este tema, solo pueden ser adecuadamente formuladas sobre la base de series mucho más largas, en las 
cuales se lleven controles cuidadosos y en que se lleven a cabo estudios planeados de antemano, más bien que simples recopilaciones de hechos cumplidos.

\section{LISTA DE REFERENCIAS EN LA LITERATURA RECIENTE}

1. Aaron, Jules B., M. D., William Levine, M. D., and Leo Gitman, M. D. Pregnancy Wastage. Obst. \& Gynec. Vol. 2. No 5. Page 461.

2. Baird, D., Prof., W. D. Winncott, M. A., FRCP, MRCS. Discussion on some Problems on Prematurity, Proceedings of the Royal Society of Medicine, Vol. 46. 1953. Page 877.

3. Bowles, H. E., The Conduct of Premature Labor. Hawai Medical Journal, May, June. 1952. Page 286.

4. Brody, Simon, M. D. and David Frenkel, M. D., Marginal Insertion of the Cord and Premature Labor. Am. J. Obst. \& Gynec.. Vol. 65, № 6. 1953, Page 1.305 .

5. Brown, Estelle W., B. S., Lyon, Robert A., M. D., and Nina A, Anderson, M. D. Causes of Prematurity. Influence of Toxemia on the Incidence of Prematurity. Am J. Dis. Child., Vol. 71, Page 378.

6. Calkins, L. A., M. D., Ph. D., Premature Spontaneous Rupture of the Membranes. Am. J. Obst. \& Gynec., Vol. 64, october 1952. Page 871.

7. Carter, Bayard, M. D., R. A. Ross, M. D., and Turner, V. H., M. D., The Prophylactic Treatment of Prematurity. North Carolina Medical Journal. Vol. 13. № 3. Page 109.

8. Cross, V. Mary, M. D., The Premature Baby - Little, Brown \& Co., 1957. Boston, Mass.

9. Csapo, Arpad, M. D., Contractility of the Uterus-Pregnancy Wastage Earl T. Engle. Editor. Vharles C. Thomas, Publisher, Springfield, III. 1953.

10. Dana, Ethel S., M. D., Premature Delivery, Causes and Results. Am. J. of Obst. \& Gynec.. Vol. 51, 1946. Page 329.

11. Drillen, Cecil Mary. The Social and Economic Factors Affecting the Incidence of Premature Birth. Part I. Premature Birth without Complications of Pregnancy. J. Obst. \& Gynec. Brit. Emp., Vol. 64. 1957. Page 161.

12. Eastman, Nicholson J., M. D., Williams Obstetrics, Ilth Edition. 1956, New York.

13. Fergusson, James H., M. D., Editorial. The Importance of Controls in a Clinical Experiment. Obst. \& Gynec., Vol. 3. № 4. 1952. Page 452.

14. Greenhill, J. P., M. D., Obstetrics, W. B. Saunders Co. 11th edition 1955.

15. Klicka, Karl S., M. D., A Home Follow-Up Plan for Care of Premature Infants, Hospitals, Vol. 25. No 7. 1951. Page 54.

16. Larson, Elizabeth, M. D., FACS. Saving the Premature Baby, Am. J. of Obst. \& Gynec., Vol. 65, № 1. Page 158.

17. Lull, Clifford B., M. D., Robert A. Kimbrough, M. D., Editors, Clinical Obstetrics, by staff members of Pennsylvanya Hospital., J. P. Lippincott.

18. Majewski, J. T., M. D., Jennings, T., M. D. A Uterine Relaxing Factor for Premature Labor. Obst. \& Gynec., Vol. 5. No 5. 1955. Page 649. 
19. Majewski, J. T., M. D., Jennings, T., M. D., Further Experiences with a Uterine Relaxing Hormone in Premature Labor. Obst. \& Gynec., Vol. 9. No 3. 1957. Page 323 .

20. Morton, Daniel G., M. D., Overstreet, Edmund W. M. D. How Can We Prevent Premature Birth. Report of a Conference. California Medicine. Vol. 77. No 1. July 1952. Page 117.

21. Mundall, R. V., N. D., Observations on Possible Relationship Between Allergic Reactions and Premature Labor. Medical Arts \& Sciences. Vol. 8. No 2. 1954. Page 54 .

22. Nesbitt, Robert E. L., Jr. M. D., Perinatal Loss in Modern Obstetrics F. A. Davis Co. publishers. 1957.

23. Ogle, Ben C., M. D., Anesthesia for Premature Labor. North Carolina Journal of Medicine. Vol. 13, No 3. Page 119.

24. Oughterson, Ashley. W., M. D., and Warren, Shields. Medical Effects of the Atomic Bomb in Japan. McGraw-Hill Book Co., Inc. 1956.

25. Points, Thomas C. M. D., Problems in Prematurity. Southern Medical Journal. Vol. 48. INay 1955. Page 541.

26. Reynolds, S. R. M., Hellman, L. M., Bruns, Paul. Dept. of Embriology Carnegie Intsitute of Washington. Patterns of Uterine Contractility in Woman during Pregnancy. The Normal and Pathological Physiology of Pregnancy. Williams \& Wilkins Co. 1948.

27. Reynolds, S. R. M., Ph. D., D. Sc., Harris, J. S., M. D., Kaiser, Irwin H. Ph. D., Clinical Measurement of Uterine Forces in Pregnancy and Labor Charles C. Thomas, Publisher. 1954.

28. Schoeneck, F. J., M. D. Obstetric Causes of Premature Labor. Obst. \& Gynec. Vol. 6. № 4. Page 444.

29. Speert, Harold, M. D., Graff, Samuel, Ph. D. and Graff, Ada M., B. A. Nutrition and Premature Labor. Am. J. of Obst. \& Gynec. Nov. 1951. Page 1.009 .

30. Tieche, Henry L., Capt., M/C., USAR., Osborn, Carl D., Capt., M. C. USAR., and Broman, John A., Capt. M. C. Is Premature Birth Preventable. Obst. \& Gynec. Vol. 7. № 5. 1956. Page 497.

31. Smith, Watkins O., Ph. D., and Smith, George Van S., M. D., Use of Diethylstilbestrol to Prevent Fetal Loss from Complications of Lage Pregnaney. New England J. of Med. Vol. 241. № 15. Page 262.

32. Whitener, Donald L., M. D., The Management of Labor and Delivery in the Interest of the Premature Infant. North Carolina Medical Journal. Vol. 13. $N^{\circ}$ 3. Page 115.

33. Tompkins, Wilson T., Wiehl, D., Nutritional Deficiences as a Causal Factor in Toxemia and Premature Labor. Am. J. of Obst. \& Gynec. Vol. 62. N 4. Page 899.

34. Annotations, Causes and Management of Premature Birth, The Lances October 1951. Page 723. 